\title{
Estimating the chance of success in IVF treatment using a ranking algorithm
}

\author{
H. Altay Güvenir ${ }^{1}$ (D) Gizem Misirli $^{1} \cdot$ Serdar Dilbaz $^{2} \cdot$ Ozlem Ozdegirmenci $^{3}$. \\ Berfu Demir ${ }^{4} \cdot$ Berna Dilbaz $^{4}$
}

Received: 29 May 2014 / Accepted: 4 April 2015 / Published online: 17 April 2015

(C) The Author(s) 2015. This article is published with open access at Springerlink.com

\begin{abstract}
In medicine, estimating the chance of success for treatment is important in deciding whether to begin the treatment or not. This paper focuses on the domain of in vitro fertilization (IVF), where estimating the outcome of a treatment is very crucial in the decision to proceed with treatment for both the clinicians and the infertile couples. IVF treatment is a stressful and costly process. It is very stressful for couples who want to have a baby. If an initial evaluation indicates a low pregnancy rate, decision of the couple may change not to start the IVF treatment. The aim of this study is twofold, firstly, to develop a technique that can be used to estimate the chance of success for a couple who wants to have a baby and secondly, to determine the attributes and their particular values affecting the outcome in IVF treatment. We propose a new technique, called success estimation using a ranking algorithm (SERA), for estimating the success of a treatment using a ranking-based algorithm. The particular ranking algorithm used here is RIMARC. The performance of the new algorithm is compared with two well-known algorithms that assign class probabilities to query instances. The algorithms
\end{abstract}

During the follow-up period of the study, Drs. Serdar Dilbaz and Ozlem Ozdegirmenci were affiliated with Etlik Zubeyde Hanim Women's Health, Teaching and Research Hospital.

H. Altay Güvenir

guvenir@cs.bilkent.edu.tr

1 Computer Engineering Department, Bilkent University, 06800 Ankara, Turkey

2 Department of Obstetrics and Gynecology, Duzce University School of Medicine, 81260 Duzce, Turkey

3 Zekai Tahir Burak Women's Health Education and Research Hospital, 06230 Ankara, Turkey

4 Etlik Zubeyde Hanim Women's Health, Teaching and Research Hospital, 06010 Ankara, Turkey used in the comparison are Naïve Bayes Classifier and Random Forest. The comparison is done in terms of area under the ROC curve, accuracy and execution time, using tenfold stratified cross-validation. The results indicate that the proposed SERA algorithm has a potential to be used successfully to estimate the probability of success in medical treatment.

Keywords Estimation of success · Ranking · Classification $\cdot$ In vitro fertilization $\cdot$ Clinical decision support system

\section{Introduction}

Assisted reproductive technologies (ART) give infertile couples a chance to have a baby. The first baby born using in vitro fertilization (IVF) was in 1978. Since 1978, different techniques, including intracytoplasmic sperm injection, pre-implantation genetic diagnosis, gamete and embryo cryopreservation, have been used as new treatment options for clinicians to achieve greater success.

Widespread use of the Internet provides information to infertile couples and raises their awareness of treatment options. Couples with infertility problems want to know their chances of having a baby when selecting the best treatment options that are based on their underlying pathology. Since the cost of IVF treatment per cycle is very high, estimating the chance of success rate per treatment cycle by using patients' personal parameters constitutes a great advantage in the field of reproductive medicine.

Machine learning techniques can be used to analyze a clinical database, including patient characteristics, all available data for ovarian hyperstimulation and pregnancy outcome. The models learned by these techniques can be used to estimate the probability of success for an infertile couple. 
In this paper, we propose a new technique, called SERA, for success estimation using ranking algorithms, which can be used for estimating the probability of success for a treatment cycle. The SERA algorithm can be used with any ranking algorithm that assigns each instance a score value to be used for ranking. We exemplify the proposed method using a dataset collected from IVF treatment records in a hospital. For a new patient couple, such a ranking method assigns a score to the couple and determines its rank among the training instances. Then, the chance of the treatment success for the new couple can be estimated as the ratio of successful training instances among those with similar score values.

The SERA algorithm, proposed in this study, is implemented using the RIMARC (ranking instances by maximizing the area under ROC curve) algorithm [11]. There are several reasons for choosing the RIMARC algorithm for SERA. Firstly, the model constructed by the RIMARC algorithm is a set of human interpretable rules that indicate the attributes and their particular values affecting the outcome. Therefore, the medical experts can validate the risk factors affecting the outcome in the IVF treatment. Another important characteristic of RIMARC is that it has no parameters that need to be tuned for the optimum performance. Therefore, there is no need for a parameter optimization step after the edition of new training data. Finally, the RIMARC algorithm is robust to missing feature values; in that, it does not require imputation of artificial values nor removal of cases or features with missing values; and it uses whatever data are available.

In IVF treatment, couples would like to know the chance of success considering their underlying pathology. An infertile couple would like to decide whether to go on with treatment considering the chance and the risks they are willing to take. We measure the success of such a chance estimation algorithm mainly in terms of AUC (area under ROC curve). We compared SERA with some wellknown algorithms that assign class probabilities to query instances. The algorithms used in the comparison are Naïve Bayes Classifier [8] and Random Forest [2]. The comparison is done in terms of both AUC and accuracy, using tenfold stratified cross-validation. The results indicate that the proposed SERA algorithm is successful in estimating the probability of success in a medical treatment.

Another aim of this study is to determine the attributes and their particular values that affect the outcome of an IVF treatment. This is done by executing the RIMAC algorithm using the whole cohort as the training data. RIMARC learns a rule for each feature. The rules are in a form interpretable by experts in the domain. These results help the experts to validate the model constructed for predicting the outcome.

The remainder of the paper is organized as follows: Sect. 2 introduces the IVF dataset used in this study. In Sect. 3, the proposed SERA algorithm is described. The
SERA algorithm is compared with four well-known classification algorithms. The results of the experiments and some of the rules learned by RIMARC are given in Sect. 4 . These rules and related work are discussed in Sect. 5. Finally, Sect. 6 concludes with future research directions.

\section{Dataset}

A dataset of 1456 patients has been compiled by the IVF Unit at Etlik Zubeyde Hanim Women's Health, Teaching and Research Hospital, located in Ankara, Turkey. This study was approved by the Local Education Planning Committee of the hospital. For each patient, the dataset contains demographic and clinical parameters, as independent features. We formed the dataset for the experiments by taking only the features that are potentially relevant for the algorithms. The dataset has one dependent feature, called Result, that has the value $P$ (for success) if the woman had a clinical pregnancy which is defined as the detection of fetal heart beat on the ultrasound examination, and the value $N$ (for failure) if the patient had only a chemical pregnancy or no pregnancy, at all. The number of $P$ labeled cases is 423 , and the $N$ labeled cases is 1033 . Therefore, the default accuracy is 0.709 , predicting the class label of all query instances as $N$.

The IVF dataset contains only the clinical features that are known before making a decision to proceed with the IVF treatment. The dataset contains 64 independent features; 52 of them are related to the female, and 12 are related to the male. The independent features included in the IVF dataset are summarized in Table 1. Among the independent features, 43 of them take on categorical values and 21 of them are numerical. Categorical features are indicated with a (C), binary (categorical feature with only two values) ones with a (B) and numerical ones with a (N). Features that take on only binary values, such as Yes/No or True/False, are treated as categorical. About $13.5 \%$ of the feature values in the dataset are missing.

\section{Proposed estimation algorithm}

The proposed success estimation algorithm in this study, SERA, uses RIMARC as its ranking algorithm. Therefore, we first sketch the RIMARC algorithm with an example of computing the score for a patient couple, and then the SERA algorithm is described.

\subsection{The RIMARC algorithm}

RIMARC is a supervised algorithm that learns a scoring function to rank instances [11]. It does not make any assumptions about the data and has no parameters to tune 
Table 1 Features in IVF dataset (N: numeric, C: Categorical, B: Binary)

\begin{tabular}{|c|c|c|}
\hline \multicolumn{2}{|l|}{ Variables related to female } & \multirow{2}{*}{$\frac{\text { Variables related to male }}{\text { Male_Factor (B) }}$} \\
\hline Female_Age (N) & Laparoscopy (C) & \\
\hline Female_Blood_Type (C) & Hysteroscopy (C) & Male_Age (N) \\
\hline Height $(\mathrm{N})$ & Laparoscopic_Surgery (C) & Male_Blood_Type (C) \\
\hline Weight $(\mathrm{N})$ & Hysteroscopic_Surgery (C) & Male_Genital_Surgery (C) \\
\hline $\mathrm{BMI}^{*}(\mathrm{~N})$ & Abdominal_Surgery (C) & Semen_Analysis_Category (C) \\
\hline Tubal_Factor (B) & Abdominal_Surgery_Category (C) & Male_FSH (N) \\
\hline Age_Related_Infertility (B) & Gynecologic_Surgery (C) & Sperm_Count (N) \\
\hline Ovulatory_Dysfunction (B) & Ovarian_Surgery $(\mathrm{C})$ & Sperm_Motility(N) \\
\hline Unexplained_Infertility (B) & Tubal_Surgery (C) & Total_Progressive_Sperm_Count (N) \\
\hline Severe_Pelvic_Adhesion (B) & Uterine_Surgery (C) & Sperm_Morphology (N) \\
\hline Endometriosis (B) & Duration_Infertility (N) & Testicular_Biopsy (C) \\
\hline Cycle_No. (N) & PCOS* (B) & TESE*_Outcome (C) \\
\hline Baseline_FSH* $(\mathrm{N})$ & HSG*_Cavity (C) & Male_Karyotype (C) \\
\hline Baseline_LH* $(\mathrm{N})$ & HSG*_Tubes* (C) & \\
\hline Baseline_E2* $(\mathrm{N})$ & Hydrosalpinx (C) & \\
\hline $\mathrm{G}^{*}(\mathrm{~N})$ & Office_Hysteroscopy(C) & \\
\hline$A^{*}(N)$ & Office_Hysteroscopic_Incision (B) & \\
\hline $\mathrm{Y}^{*}(\mathrm{~N})$ & Office_Hysteroscopic_Procedure (C) & \\
\hline $\mathrm{DM}^{*}(\mathrm{C})$ & Total_Antral_Follicle_Count (N) & \\
\hline $\mathrm{HT}^{*}(\mathrm{~B})$ & Right_Ovarian_Antral_Follicle_Count (N) & \\
\hline Thyroid_Disease (C) & Left_Ovarian_Antral_Follicle_Count (N) & \\
\hline Anemia (B) & Hyperprolactinemia (B) & \\
\hline Laparotomy (C) & Hepatitis (C) & \\
\hline Cyst_Aspiration (B) & Endometrioma_Surgery (C) & \\
\hline Embryocryo (B) & Localization_Myoma_Uteri (C) & \\
\hline
\end{tabular}

* BMI body mass index, $F S H$ follicle-stimulating hormone, $L H$ luteinizing hormone, $E 2$ estradiol, $G$ gravida, $A$ abortus, $Y$ living children, $D M$ diabetes mellitus, $H T$ hypertension, $P C O S$ polycystic ovary syndrome, $H S G$ hysterosalpingography, TESE testicular sperm extraction

for optimizing the performance. The RIMARC algorithm aims to maximize the AUC value, since the area under the ROC curve (AUC) has become a widely accepted performance evaluation metric in evaluating the quality of ranking. It learns a ranking function which is a linear combination of nonlinear score functions constructed separately for each feature. Each of these nonlinear score functions aims to maximize the AUC by considering only the corresponding feature in ranking. It has been shown that, for a single categorical feature, it is possible to derive a scoring function that achieves the maximum possible AUC. Therefore, the RIMARC algorithm first discretizes all continuous features into categorical ones, in a way that optimizes the AUC, by using the MAD2C algorithm proposed by Kurtcephe and Güvenir [18].

A categorical feature $f$ has a finite set of values. Let $V_{f}=\left\{v_{1}, v_{2}, \ldots, v_{\mathrm{k}}\right\}$ be the set of values for a given categorical feature $f$. Consider a dataset that includes only this feature and a class value for each instance. That is, an instance is represented by two values: $f$ value and class label. A scoring function $s_{f}()$ can be defined to rank the elements of $V_{f}$. According to this scoring function, $v_{\mathrm{i}} \preccurlyeq v_{\mathrm{j}}$ if and only if $s_{f}\left(v_{\mathrm{i}}\right) \leq s_{f}\left(v_{\mathrm{j}}\right)$. Note that the problem of ranking the instances in a dataset is reduced to the problem of ranking the values of a feature. Güvenir and Kurtcephe showed that a scoring function has to satisfy the following condition in order to achieve the maximum AUC [11]:

$s_{f}\left(v_{\mathrm{i}}\right) \leq s_{f}\left(v_{\mathrm{j}}\right)$ iff $\frac{P_{\mathrm{i}}}{N_{\mathrm{i}}}<\frac{P_{\mathrm{j}}}{N_{\mathrm{j}}}$

Here $P_{\mathrm{i}}$ is the number of positive ( $P$ labeled) instances and $N_{\mathrm{i}}$ is the number of negative ( $N$ labeled) instances, for the value $v_{\mathrm{i}}$ of feature $f$. Note that any scoring function that satisfies this condition will result in the maximum possible AUC in ranking the dataset with a single feature. It is important to note that, for some values of $i, N_{\mathrm{i}}$ may be 0 . In such cases, the ranking function will have an undefined value. In order to overcome this problem, the RIMARC algorithm defines the ranking function as follows:

$s_{f}\left(v_{\mathrm{i}}\right)=\frac{P_{\mathrm{i}}}{P_{\mathrm{i}}+N_{\mathrm{i}}}$ 
Table 2 An example of computing score using RIMARC

\begin{tabular}{lllll}
\hline Feature & Feature weight $w_{f}$ & Feature value & Score value $s_{f}(q)$ & Weighted score $w_{f} s_{f}(q)$ \\
\hline Female_Age & 0.1753 & 25 & 0.2375 & 0.0416 \\
BMI & 0.1443 & 25.7 & 0.2169 & 0.0313 \\
Semen_Analysis_Category & 0.1407 & Astheno & 0.3571 & 0.0503 \\
Age_Related_Infertility & 0.1178 & No & 0.2245 & 0.0264 \\
Sum & $\mathbf{0 . 5 7 8 1}$ & & & $\mathbf{0 . 1 4 9 6}$ \\
Score $(q)=0.1496 / 0.5781$ & $\mathbf{0 . 2 5 8 7}$ & & & \\
\hline
\end{tabular}

Bold values indicate the results
This newly defined scoring function satisfies the condition in Eq. (1) and furthermore is interpretable by medical doctors, since it is simply the probability of the $p$ label among all instances with value $v_{\mathrm{i}}$. This probability value is easily interpretable by humans. The instances of the dataset, which has a single categorical feature $f$, are sorted by the scoring function $s_{f}()$, and the AUC is computed. The AUC obtained by this scoring function is guaranteed to be between 0.5 and 1.0 [11]. If the feature $f$ is irrelevant, the AUC will be 0.5 . On the other hand, if the single feature $f$ is sufficient to predict the class label, all positive and negative instances will be separated by the scoring function $s_{f}()$, and the AUC will be 1.0. The RIMARC algorithm uses the AUC value to measure the weight (relevancy) of the feature $f$ as:

$w_{f}=2\left(\mathrm{AUC}_{f}-0.5\right)$,

where $\mathrm{AUC}_{f}$ is the AUC obtained for feature $f$. Therefore, the weight of a feature will be in the range of $[0,1]$. The RIMARC algorithm computes the weight of each feature by setting up a sub-dataset, which is composed of only that feature and the class.

As an example, suppose that the AUC computed for the feature $f$ is 1 . This means perfect ordering, and this is the maximum value that AUC can have. That is, all instances in the training set can be ranked by using only the values of feature $f$. Therefore, we expect that query instances can be ranked correctly among the training set by using only feature $f$.

The rule model learned by the RIMARC algorithm is used to compute the score for a given query patient $q$ as:

$\operatorname{score}(q)=\frac{\sum_{f} w_{f}^{q} \cdot s_{f}(q)}{\sum_{f} w_{f}^{q}}$

$w_{f}^{q}= \begin{cases}w_{f} & q_{f} \text { is known } \\ 0 & q_{f} \text { is missing }\end{cases}$

Here $w_{f}$ represents the weight of the feature $f$, and $s_{f}(q)$ represents the score associated with the value of feature $f$ for patient couple $q$, queried. The RIMARC algorithm is robust to missing feature values. The features whose values in query $q$ are missing are simply ignored when computing the score for that query. For example, consider a 25-year-old female, whose BMI is 25.7, she does not have age-related infertility, the semen analysis category for her partner is asthenospermia, and the values of all other features are missing. Then the score of the treatment for this couple can be computed as shown in Table 2 .

In summary, the RIMARC ranking algorithm does not have parameters that have to be tuned after the addition of new records about existing or new patients. Further, the ranking knowledge constructed by RIMARC includes information about the importance of features as weights and effects of particular values or ranges in the success of the treatment in terms of scores. This form of knowledge can be analyzed and verified by the domain experts.

Another important characteristic of the REMARC algorithm is its robustness to missing feature values. Since it processes each feature individually, missing feature values are simply ignored when processing the corresponding feature. Therefore, instead of ignoring a complete patient record with missing feature values, or imputing them with artificial values, it uses all data available about a patient.

\subsection{The SERA algorithm}

The SERA algorithm is designed to estimate the chance of a treatment as the probability of success of the treatment for a patient couple. It uses the score assigned to the couple by a ranging algorithm to determine the similar past cases. Although it can be any ranking algorithm that assigns score values for the instances, it uses the RIMARC algorithm for the reasons given above. The ranking score value is used to locate the query patient among the training cases. However, what we need is the chance of success for a new infertile couple. On the other hand, semantically, the word "chance" refers to the probability. In order to calculate the chance of success of IVF treatment for a query patient $q$, we select the first $k$ (e.g., $k=100$ ) past (training) patients whose ranking scores are closest to $\operatorname{score}(q)$, the score computed by the query couple. If the number of successful cases among these $k$ similar training cases is $P_{\text {count }}$, then the chance of success for $q$ is reported as

$\operatorname{chance}(q)=\frac{P_{\text {count }}}{k}$

That is, chance $(q)$ represents the probability of success considering the most similar $k$ past cases in terms of 


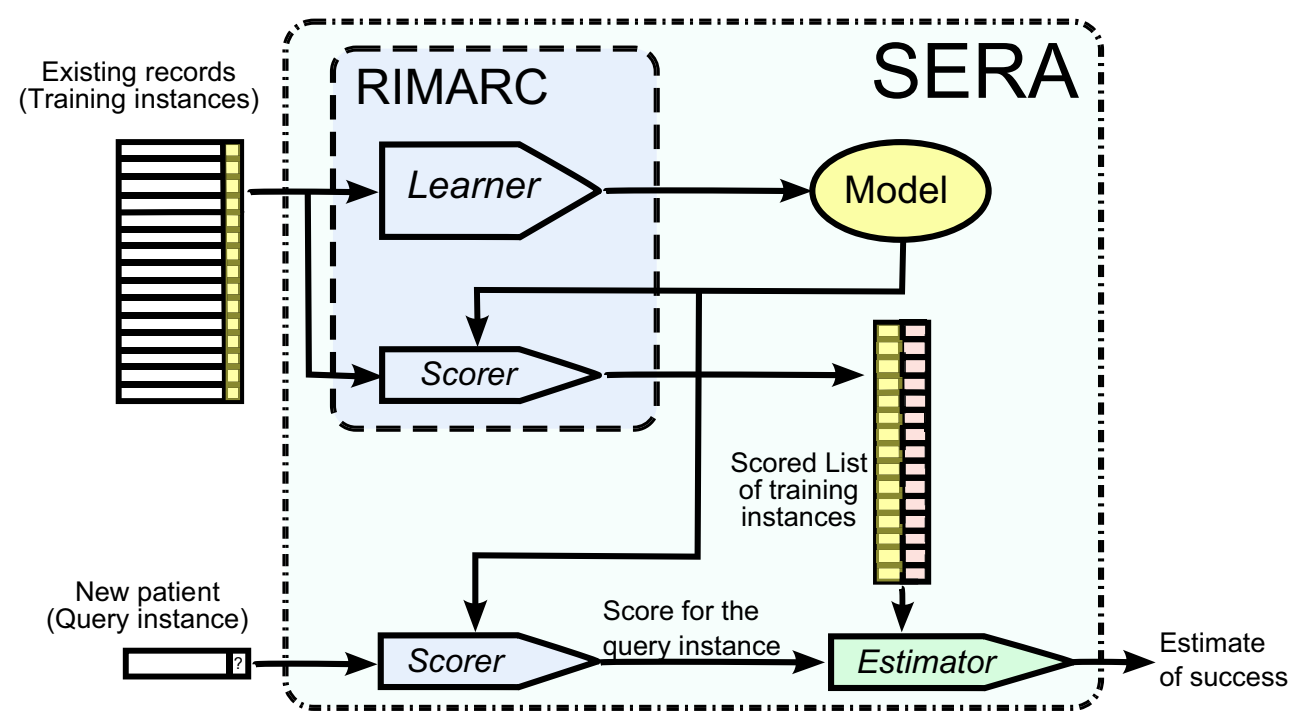

Fig. 1 Block diagram of the SERA algorithm

the score values. An alternative approach, to determine the neighbors, is to fix the radius $r$, and select all training instances $p$, such that $|\operatorname{score}(q)-\operatorname{score}(p)|<r$, as the neighbors of $q$.

It should be noted that the chance $(q)$ is the probability among the past instances with similar score values. In reality, one would be interested in patient similarity in terms of metrics such as Euclidean distance. Similar instances have similar score values, whereas instances with similar score values may look different. This might appear to be a limitation. However, using a successful scoring function, it leads to efficient searches for similar cases. The block diagram of the SERA algorithm is given in Fig. 1.

The SERA algorithm can also be used for binary classification, where the class labels are $P$ and $N$. As shown in Eq. (6), the class label of a query instance $q$ can be predicted as $P$ if the chance $(q)$ is higher than $50 \%$.

$\operatorname{prediction}(q)= \begin{cases}P & \text { chance }(q)>0.5 \\ N & \text { otherwise }\end{cases}$

\section{Results}

In this section, we present a comparison of the SERA algorithm with some well-known classification algorithms that assign class probabilities to query instances. The algorithms used in the comparison are Naïve Bayes Classifier [8] and Random Forest [2]. Despite their simplicity, Naïve Bayes Classifiers have worked well in many complex real-world situations. Further, they are suited when the dimensionality of the inputs is high. The choice of Random Forest is due to the fact that they cope with the problem of overfitting the training data by averaging multiple deep decision trees, trained on different parts of the same training set, with the goal of reducing the variance [13].

The comparison is performed in terms of area under the ROC curve and accuracy, using tenfold stratified cross-validation, over 200 random datasets obtained by shuffling the original dataset. Tests for these algorithms are performed using the Weka toolbox, which is a collection of machine learning algorithms, implemented in the Java language [12]. The Naïve Bayes Classifier and Random Forest algorithms are implemented as java classes as Naive Bayes and Random Forest. These classes replace all missing values for nominal and numeric attributes in a dataset with the modes and means from the training data, respectively. In our experiments, all parameters for the algorithms are set to their default values. Although, in general, classification algorithms are badly affected from the curse of dimensionality, we did not apply any technique to reduce the number of features, since one of the aims of this study is to determine the attributes and their particular values that affect the outcome of an IVF treatment.

The SERA algorithm is also implemented in the Java language. The number of nearest neighbors considered by SERA is set to 100 (default). Table 3 displays the results of the experiments ${ }^{1}$ conducted using the IVF dataset. As seen from the table, SERA outperforms the other classifiers in terms of both AUC and accuracy. Also SERA is the second fasted algorithm in terms of the execution time.

We further experimented with the choice of $k$ (number of similar instances considered) on the AUC and accuracy with the IVF dataset. Both AUC and accuracy remain

\footnotetext{
${ }^{1}$ Experimented are performed on a PC with 64-bit Windows 7 OS, $3 \mathrm{GHz}$, Core2Duo CPU and 4 GB RAM.
} 
Table 3 Results of AUC, accuracy and execution time on the IVF dataset

\begin{tabular}{|c|c|c|c|c|c|c|}
\hline \multirow[t]{3}{*}{ Algorithm } & \multirow[t]{3}{*}{ AUC } & \multicolumn{3}{|c|}{ Area under ROC curve } & \multirow[t]{3}{*}{ Accuracy } & \multirow{3}{*}{$\begin{array}{l}\text { Execution } \\
\text { time (s) }\end{array}$} \\
\hline & & \multirow[t]{2}{*}{ SE } & \multicolumn{2}{|c|}{$95 \%$ Confidence interval } & & \\
\hline & & & Lower bound & Upper bound & & \\
\hline SERA & $0.833( \pm 0.003)$ & 0.012 & $0.809( \pm 0.003)$ & $0.857( \pm 0.004)$ & $0.844( \pm 0.004)$ & $1.4( \pm 0.2)$ \\
\hline NBC & $0.794( \pm 0.002)$ & 0.014 & $0.767( \pm 0.002)$ & $0.822( \pm 0.002)$ & $0.783( \pm 0.002)$ & $0.8( \pm 0.1)$ \\
\hline Random forest & $0.769( \pm 0.009)$ & 0.014 & $0.741( \pm 0.010)$ & $0.797( \pm 0.008)$ & $0.792( \pm 0.008)$ & $2.0( \pm 0.1)$ \\
\hline
\end{tabular}

The values are mean $( \pm \mathrm{SD})$ over 200 runs with random shuffling of the dataset $(p<0.0005$ for all algorithms) almost constant for values $k>40$. The accuracy slightly drops when $k>230$. The graph is shown in Fig. 2.

Another aim of this study is to determine the attributes and their particular values that affect the outcome of an IVF treatment. This is done by executing the RIMAC algorithm using the whole cohort as the training data. RIMARC learns a rule for each feature. The rules are in form interpretable by experts in the domain. These rules help the experts to validate the model constructed for predicting the outcome. The feature weights learned by RIMARC, using the whole cohort as the training set, are shown in Table 4. For continuous attributes, the threshold values over or below which the chances of success change drastically are very valuable. The RIMARC algorithm determines these threshold values by discretizing the continuous attributes using the MAD2C algorithm [18]. Some of the rules learned for some of the features, using the whole cohort as the training set to RIMARC, are shown in Fig. 3.

Reproductive aging occurs as a consequence of the decrease in the quantity and quality of the ovarian follicles [34]. Approximately 1000 follicles are depleted per month during the reproductive period, and this depletion increases significantly after the age of 35 [9]. The antral follicle count is a reliable diagnostic test for the evaluation of ovarian reserve. Ovarian reserve tests reflect the quantitative aspect of the ovarian reserve status. These tests are mainly used to predict the response of the ovarian hyperstimulation. However, prediction of the success of IVF treatment is mainly based on the quality of the oocytes. Recently, a meta-analysis by Broer et al. [3] assessed the additional value of the ovarian reserve test in predicting IVF success. According to the results of the RIMARC, female age and total antral follicle count are significant predictors of IVF success. Clinical pregnancy rates significantly decrease after the age of 34.5 and when the total antral follicle count falls below 10.5 .

The impact of obesity on the outcome of infertility treatment is a contentious issue. While some studies associate obesity with the need for higher doses of gonadotropins, increased cycle cancellation rates, fewer oocyte yield and higher miscarriage rates $[10,24,32]$, other studies have

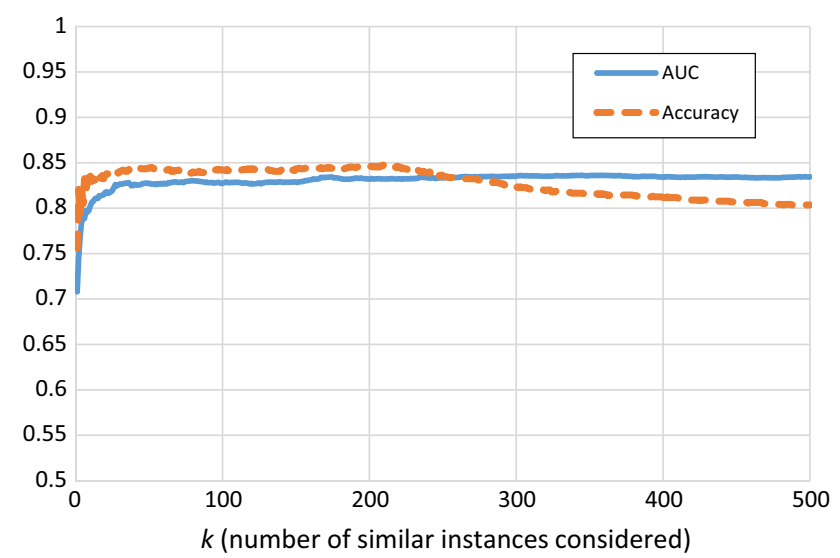

Fig. 2 Effect of $k$ on AUC and accuracy

been unable to find any negative effect of obesity on IVF outcome [7, 20]. In experiments with RIMARC, an important decrease in the clinical pregnancy rate was observed when the BMI was higher than 27.9.

\section{Discussion}

Infertility is defined as the failure to conceive after 12 months or more of regular unprotected intercourse [29]. In 2010, among women 20-44 years of age who were exposed to the risk of pregnancy, $1.9 \%$ were unable to attain a live birth (primary infertility) [25]. Of the women who had had at least one live birth and were exposed to the risk of pregnancy, $10.5 \%$ were unable to have another child (secondary infertility). The major causes of infertility are as follows: ovulatory dysfunction (15\%), tubal and peritoneal pathology $(35 \%)$, male infertility $(35 \%)$, unexplained infertility $(10 \%)$ and unusual reasons $(5 \%)[4,15]$.

IVF involves a sequence of coordinated procedures that begin with controlled ovarian hyperstimulation $(\mathrm{COH})$, followed by retrieval of oocytes from the ovaries under the guidance of transvaginal ultrasonography, fertilization of the oocytes and spermatozoa at the embryology laboratory, and finally, embryo transfer into the uterine cavity. 
Table 4 Feature weights learned by RIMARC

\begin{tabular}{|c|c|c|c|}
\hline Feature & Weight & Feature & Weight \\
\hline Laparoscopic_Surgery & 0.6455 & Laparotomy & 0.0909 \\
\hline Total_Antral_Follicle_Count & 0.5498 & Male_Karyotype & 0.0834 \\
\hline Right_Ovarian_Antral_Follicle_Count & 0.5163 & HSG_Tubes & 0.0783 \\
\hline Left_Ovarian_Antral_Follicle_Count & 0.4934 & Myoma_Uteri & 0.0737 \\
\hline Hysteroscopic_Surgery & 0.457 & Uterine_Surgery & 0.0711 \\
\hline TESE_Outcome & 0.4254 & Sperm_Morphology & 0.0708 \\
\hline Female_Age & 0.3957 & Abdominal_Surgery & 0.053 \\
\hline Male_FSH & 0.3484 & Cycle_No & 0.0508 \\
\hline Male_Blood_Type & 0.3118 & Tubal_Factor & 0.0496 \\
\hline Male_Age & 0.2777 & Cyst_Aspiration & 0.0384 \\
\hline Baseline_FSH & 0.2764 & Ovarian_Surgery & 0.0354 \\
\hline PCOS & 0.2258 & Male_Factor & 0.0332 \\
\hline Total_Progressive_Sperm_Count & 0.2151 & Endometrioma_Surgery & 0.0276 \\
\hline Sperm_Count & 0.2098 & Abdominal_Surgery_Category & 0.0274 \\
\hline Localization_Myoma_Uteri & 0.2021 & Thyroid_Disease & 0.0273 \\
\hline Age_Related_Infertility & 0.1959 & Testicular_Biopsy & 0.0256 \\
\hline Ovulatory_Dysfunction & 0.1791 & Laparoscopy & 0.0231 \\
\hline Gynecologic_Surgery & 0.1779 & Hysteroscopy & 0.0197 \\
\hline Semen_Analysis_Category & 0.1777 & $\mathrm{DM}$ & 0.0141 \\
\hline Unexplained_Infertility & 0.1775 & Tubal_Surgery & 0.0125 \\
\hline Duration_Infertility & 0.1567 & HT & 0.0122 \\
\hline BMI & 0.1534 & $\mathrm{Y}$ & 0.012 \\
\hline Height & 0.1339 & Endometriosis & 0.0118 \\
\hline Weight & 0.1333 & Embryocryo & 0.0117 \\
\hline Female_Blood_Type & 0.127 & Hydrosalpinx & 0.0104 \\
\hline Office_Hysteroscopic_Procedure & 0.1245 & G & 0.0101 \\
\hline Office_Hysteroscopy & 0.1238 & Office_Hysteroscopic_Incision & 0.01 \\
\hline Baseline_LH & 0.1196 & A & 0.0093 \\
\hline HSG_Cavity & 0.1048 & Hyperprolactinemia & 0.0085 \\
\hline Male_Genital_Surgery & 0.1039 & Hepatitis & 0.0079 \\
\hline Sperm_Motility & 0.096 & Severe_Pelvic_Adhesion & 0.0047 \\
\hline Baseline_E2 & 0.095 & Anemia & 0.0004 \\
\hline
\end{tabular}

Controlled ovarian hyperstimulation is used to induce the growth of multiple follicles. Numerous treatment regimens have been described; the most preferred agents are gonadotropins in combination with a gonadotropin-releasing hormone (GnRH) agonists or antagonists. GnRH agonists or antagonists are mainly used to prevent a premature $\mathrm{LH}$ surge. $\mathrm{COH}$ protocols are described according to the use of oral contraceptives, timing and duration of $\mathrm{GnRH}$ agonists such as the long, short, micro-dose flare up, and stop protocol. The selection of the $\mathrm{COH}$ protocols in clinical practice is based mainly on the patient's age and ovarian reserve (poor or hyperresponder).

FSH-containing gonadotropins are used for ovarian stimulation. Human menopausal gonadotropins are extracted from the urine of postmenopausal women. Highly purified urinary FSH preparations with no contaminating urinary proteins are produced. Advanced technology is used to produce recombinant gonadotropins that are free of contamination of proteins and viruses. All gonadotropins are orally inactive [1]. Ovarian reserve and body mass index are important parameters in the determination of the daily gonadotropin dosage.

Oocyte retrieval is generally performed approximately 34-36 $\mathrm{h}$ after hCG administration. The standard technique of oocyte retrieval is performed under the guidance of the transvaginal ultrasonography with intravenous sedation anesthesia.

To as close as possible to the time of the oocyte retrieval, a semen sample is obtained by masturbation. If a patient has no sperm in the ejaculate (azoospermia), a variety of surgical approaches is used for sperm extraction. Microscopic testicular sperm extraction is the most complicated procedure among them. 


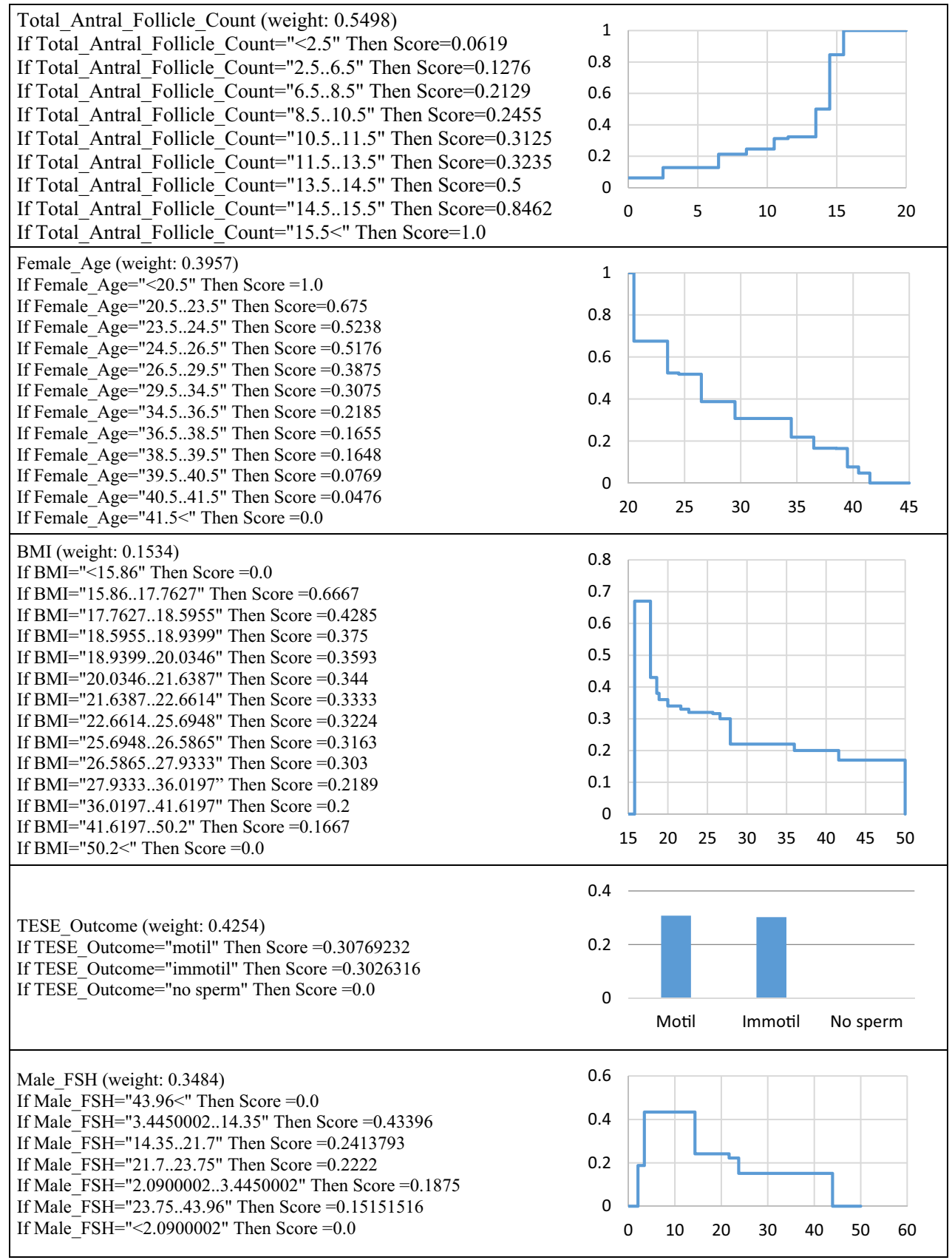

Fig. 3 Some rules learned by RIMARC

In the IVF procedure, an oocyte is incubated with 100,000-200,000 motile spermatozoa in vitro. If the selected spermatozoa is injected into the ooplasm using an injection pipette under a microscope, this procedure is called intracytoplasmic sperm injection.

The main important factors affecting the success rate of IVF are woman's age and the cause of infertility. Other prognostic factors are as follows: hydrosalpinx, uterine myomas, smoking and obesity.
Clinical pregnancy is defined as the presence of an intrauterine gestational sac with fetal cardiac activity as confirmed by transvaginal ultrasonography. Chemical pregnancy is accepted as low level of $\beta$ hCG that is not confirmed through visualization of the gestational sac.

We showed that machine learning systems could help to determine the relative weights of the risk factors and the relative effects of the particular values of these risk factors. Most importantly, machine learning systems could predict 
the personalized chance of the outcome related to these factors to help decide whether to start the complex IVF treatment procedure.

IVF treatment is a long, complex and costly process. It is very stressful for couples who want to have a baby. Although there many machine learning applications for clinical decision support systems in the literature [6, 19, 22, 27], systems related to obstetrics is limited [28]. The literature shows that in early studies, case-based reasoning systems and neural networks have been constructed to predict the outcome of IVF [16, 17]. Subsequently, decision tree models are constructed to predict the outcome of IVF treatment [33, 34]. The most recent studies on IVF propose Naive Bayes, Bayesian Classification and Support Vector Machines to increase the chance of having a baby after IVF treatment. Uyar et al. [35] studied for implantation prediction on IVF embryos using Naive Bayes classification. In another study, the embryo implantation prediction is defined. In this study, embryo-based prediction is identified in order to predict the outcome of IVF treatment and an SVM-based learning system is used [37]. In addition, there is a study related to predicting implantation potentials of IVF embryos [36]. Predicting the IVF outcome is a considerably challenging process, so much research aims to address this problem $[5,26]$.

The area under the ROC curve (AUC) is a widely accepted performance measure for evaluating the quality of ranking [21]. It has become a popular performance measure in the machine learning community after it was discovered that accuracy is often a poor metric to evaluate classifier performance $[13,14,23,30,31]$.

\section{Conclusions}

In vitro fertilization is a common infertility treatment method in which female oocytes are inseminated by sperm under laboratory conditions. Given a new candidate for IVF, the first important consideration is whether to apply the IVF treatment or not. The decision is made mainly by the clinician and the couple. Since the IVF treatment involves an application of several hormones and medicines to both female and male patients, it is a difficult and stressful process. If the chances of success are low, the couple may choose not to start the treatment. However, as in many areas of medicine it is not possible to construct a mathematical model that, given the values of relevant parameters for a couple, returns the outcome of the IVF treatment.

In this paper, we showed that it is possible to learn a model, from a set of past cases of IVF treatment, which can estimate the outcome of the treatment for a given couple. We tested three such score-based ranking algorithms, namely SERA, Naïve Bayesian Classifier and Random
Forest. These supervised machine learning algorithms applied to a dataset of cases learn a model that can be used to estimate the likelihood of success. We applied these algorithms to a dataset of cases, where each case, called a cycle, represents the values of parameters that are measured before applying the IVF treatment, along with the outcome of the treatment.

The RIMARC algorithm, used by SERA, has three important characteristics for medical applications. Firstly, it learns rules about the data, which can be further analyzed by medical practitioners. Secondly, it does not have parameters that need to be optimized after the addition of new patient records. Finally, it is robust to missing feature values, which is common in medical datasets. Further, the results of our experiments showed that the SERA algorithm outperformed the others in terms of both AUC and accuracy.

Further, the RIMARC algorithm calculates feature weights and creates rules that are in a human-readable form and easy for clinicians to interpret. This characteristic of RIMARC enables clinicians to validate the model constructed.

As a future work, we plan to collect similar datasets from other IVF clinics and apply the SERA algorithm. We will investigate whether the models agree with the one constructed in this study. If the models differ to a large extent, then the possible difference in the patient profile should be investigated. Further, we plan to apply the SERA algorithm to datasets from other disciplines of medicine.

Acknowledgments This research was funded by Bilkent University and Etlik Zubeyde Hanim Women's Health Teaching and Research Hospital.

Open Access This article is distributed under the terms of the Creative Commons Attribution 4.0 International License (http://creativecommons.org/licenses/by/4.0/), which permits unrestricted use, distribution, and reproduction in any medium, provided you give appropriate credit to the original author(s) and the source, provide a link to the Creative Commons license, and indicate if changes were made.

\section{References}

1. Aboulghar M, Botroz R (eds) (2011) Ovarian stimulation. Cambridge University Press, pp 61-66

2. Breiman L (2001) Random forests. Mach Learn 45(1):5-32

3. Broer SL, van Disseldorp J, Broeze KA, Dolleman M, Opmeer BC, Bossuyt P, Eijkemans MJ, Mol BW, Broekmans FJ (2013) Added value of ovarian reserve testing on patient characteristics in the prediction of ovarian response and ongoing pregnancy: an individual patient data approach. Hum Reprod Update 19(1):26-36

4. Collins JA, Burrows EA, Wilan AR (1995) The prognosis for live birth among untreated infertile couples. Fertil Steril 64(1):22-28

5. Corani G, Magli C, Giusti A, Gianaroli L, Gambardella L (2012) A Bayesian network model for predicting the outcome of in vitro 
fertilization. In: Proceedings of the sixth European workshop on probabilistic graphical models. Granada, Spain, pp 75-82

6. Cuaya G, Munoz Melendez A, Carrera LN, Morales EF, Quinones I, Perez AI, Alessi A (2013) A dynamic Bayesian network for estimating the risk of falls from real gait data. Med Biol Eng Comput 51(1-2):29-37

7. Dechaud H, Anahory T, Reyftmann L, Loup V, Hamamah S, Hedon B (2006) Obesity does not adversely affect results in patients who are undergoing in vitro fertilization and embryo transfer. Eur J Obstet Gynecol Reprod Biol 127:88-93

8. Duda R, Hart P (1973) Pattern classication and scene analysis. Wiley, New York

9. Faddy MJ, Gosden RG, Gougeon A et al (1992) Accelerated disappearance of ovarian follicles in mid-life: implications for forecasting menopause. Hum Reprod 7(10):1342-1346

10. Fedorcsak P, Dale PO, Storeng R, Ertzeid G, Bjercke S, Oldereid $\mathrm{N}$ et al (2004) Impact of overweight and underweight on assisted reproduction treatment. Hum Reprod 19:2523-2528

11. Güvenir HA, Kurtcephe M (2013) Ranking instances by maximizing the area under ROC curve. IEEE Trans Knowl Data Eng 25(10):2356-2366

12. Hall M, Frank E, Holmes G, Pfahringer B, Reutemann P, Witten IH (2009) The WEKA data mining software: an update. SIGKDD Explor 11(1):10-18

13. Hastie T, Tibshirani R, Friedman J (2011) The elements of statistical learning: data mining, inference, and prediction, 2nd edn. Springer, New York

14. Huang J, Ling CX (2005) Using AUC and accuracy in evaluating learning algorithms. IEEE Trans Knowl Data Eng 17(3):299-310

15. Hull MG, Glazener CM, Kelly NJ, Conway DI, Foster PA, Hinton RA, Coulson C, Lambert PA, Watt EM, Desai KM (1985) Population study of causes, treatment, and outcome of infertility. Br Med J 291(6510):1693-1697

16. Jurisica I, Mylopoulos J, Glasgow J, Shapiro H, Casper RF (1998) Case-based reasoning in IVF: prediction and knowledge mining. Artif Intell Med 12(1):1-24

17. Kaufmann SJ, Eastauh JL, Snowden S, Smye SW, Sharma V (1997) The application of neural Networks in predicting the outcome of in vitro fertilization. Hum Reprod 12(7):1454-1457

18. Kurtcephe M, Güvenir HA (2013) A discretization method based on maximizing the area under ROC curve. J Pattern Recognit Artif Intell 27(1):1350002

19. Kim KA, Choi JY, Yoo TK, Kim SK, Chung K, Kim WD (2013) Mortalilty prediction of rats in acute hemorrhagic shock using machine learning techniques. Med Biol Eng Comput 51:1059-1067

20. Lashen H, Ledger W, Bernal AL, Barlow D (1999) Extremes of body mass do not adversely affect the outcome of superovulation and in vitro fertilization. Hum Reprod 14:712-715

21. Lasko TA, Bhagwat JG, Zou KH, Ohno-Machado L (2005) The use of receiver operating characteristic curves in biomedical informatics. J Biomed Inform 38(5):404-415

22. Liu NT, Holcomb JB, Wade CE, Batchinsky AI, Cancio LC, Darrah MI, Salinas J (2014) Development and validation of a machine learning algorithm and hybrid system to predict the need for life-saving interventions in trauma patients. Med Biol Eng Comput 52:193-203
23. Logesparan L, Casson AJ, Rodriguez-Villegas E (2012) Optimal features for online seizure detection. Med Biol Eng Comput 50(7):659-669

24. Maheshwari A, Stofberg L, Bhattacharya S (2007) Effect of overweight and obesity on assisted reproductive technology-a systematic review. Hum Reprod Update 13:433-444

25. Mascarenhas MN, Flaxman SR, Boerma $T$, Vanderpoel $S$, Gretchen A, Stevens GA (2012) National, regional, and global trends in infertility prevalence since 1990: a systematic analysis of 277 health surveys. PLOS Med 9(12):e1001356

26. Morales DA, Bengoetxea E, Larranaga B, Garcia M, Franco Y, Fresnada M, Merino M (2008) Bayesian classification for the selection of in vitro human embryos using morphological and clinical data. Comput Methods Programs Biomed 90(2):104-116

27. Oshiyama NF, Bassani RA, D’Ottaviano IML, Bassani JWM (2012) Medical equipment classification: method and decisionmaking support based on paraconsistent annotated logic. Med Biol Eng Comput 50:395-402

28. Potočnik B, Cigale B, Zazula D (2012) Computerized detection and recognition of follicles in ovarian ultrasound images: a review. Med Biol Eng Comput 50(12):1201-1212

29. Practice Committee of the American Society for Reproductive Medicine (2013) Definitions of infertility and recurrent pregnancy loss: a committee opinion. Fertil Steril 99(1):63

30. Provost F, Fawcett T (1997) Analysis and visualization of classifier performance: comparison under imprecise class and cost distributions. In: Proceedings of the third international conference on knowledge discovery and data mining. AAAI Press, pp 43-48

31. Provost F, Fawcett T, Kohavi R (1998) The case against accuracy estimation for comparing induction algorithms. In: Proceedings of the fifteenth international conference on machine learning. Morgan Kaufmann, pp 445-453

32. Rittenberg V, Seshadri S, Sunkara SK, Sobaleva S, Oteng-Ntim E, El-Toukhy T (2011) Effect of body mass index on IVF treatment outcome: an updated systematic review and meta-analysis. Reprod Biomed Online 23:421-439

33. Saith R, Srinivasan A, Michie D, Sargent I (1998) Relationships between the developmental potential of human in vitro fertilization embryos and features describing the embryo, oocyte and follicle. Hum Reprod Update 4(2):121-134

34. Te Velde ER, Pearson PL (2002) The variability of female reproductive ageing. Hum Reprod Update 8:141-154

35. Uyar A, Bener A, Çıray N, Bahçeci M (2009) Predicting implantation outcome from imbalanced IVF dataset. In: Ao SI, Douglas C, Grundfest WS, Burgstone J (eds) Proceedings of the World Congress on Engineering and Computer Science, Vol II Oct. 20-22, 2009, San Francisco, USA, Newswood Limited

36. Uyar A, Bener A, Ciray H, Bahceci M (2010) ROC based evaluation and comparison of classifiers for IVF implantation prediction. In: Kostkova P (ed) Lecture Notes of the Institute for Computer Sciences, Social Informatics and Telecommunications Engineering, vol 27. Springer, Berlin, pp 108-111

37. Uyar A, Ciray HN, Bener A, Bahceci M (2009) 3P: personalized pregnancy prediction in IVF treatment process. Electron Healthc 0001:58-65 